VoL. $68(2003)$ [213-219]

\title{
OPEN ENGEL MANIFOLDS ADMITTING COMPACT CHARACTERISTIC LEAVES
}

\author{
TAKASHI INABA
}

\begin{abstract}
We give an example of an Engel structure on the 4-dimensional Euclidean space which admits a compact characteristic leaf. We also show that every Engel structure on an open 4-manifold can be modified so that the resulting structure has a compact characteristic leaf.
\end{abstract}

\section{INTRODUCTION}

An Engel structure is a 2-plane field on a 4-manifold which is "most non-integrable". Given an Engel structure $\mathcal{D}$, there uniquely exists a certain line field $\mathcal{L}(\mathcal{D})$ tangent to $\mathcal{D}$, called the characteristic line field of $\mathcal{D}$. We are interested in how rich the dynamics of $\mathcal{L}(\mathcal{D})$ can be. In this paper we mainly consider Engel structures on the 4-dimensional Euclidean space $\mathbf{R}^{4}$. For the standard Engel structure $\mathcal{D}_{0}$ on $\mathbf{R}^{4}$, the leaves (integral curves) of the characteristic line field are parallel straight lines. In [3] and [4], Gershkovich modified $\mathcal{D}_{0}$ in several ways and produced some "exotic" Engel structures on $\mathbf{R}^{4}$. But none of them has a compact characteristic leaf. So, in [3], he posed the following question: Does there exist an Engel structure on $\mathbf{R}^{4}$ whose characteristic line field has a compact leaf? In this paper we shall answer the question by constructing a simple concrete example. We also show that by a similar construction we can modify a given Engel structure on an open 4-manifold so as to have a compact characteristic leaf.

\section{Statement of the Result}

Let $M$ be a 4-dimensional manifold and $\mathcal{D}$ a 2-plane field on $M$. Then $\mathcal{D}$ is an Engel structure on $M$ if the Lie bracket $\mathcal{D}^{2}=[\mathcal{D}, \mathcal{D}]$ is a 3-plane field and $\left[\mathcal{D}, \mathcal{D}^{2}\right]=T M$. (Here, we have identified a plane field with the sheaf of local vector fields which are tangent to the plane field.) A manifold with an Engel structure is called an Engel manifold.

Received 13th January, 2003

Partially supported by Grant-in-Aid for Scientific Research C2, No.14540064, Ministry of Education, Science, Sports and Culture, Japan.

Copyright Clearance Centre, Inc. Serial-fee code: 0004-9727/03 \$A2.00+0.00. 
Any Engel structure $\mathcal{D}$ has a canonical line field $\mathcal{L}(\mathcal{D}) \subset \mathcal{D}$ characterised by the property that $\left[\mathcal{L}(\mathcal{D}), \mathcal{D}^{2}\right]$ is contained in $\mathcal{D}^{2}$. The line field $\mathcal{L}(\mathcal{D})$ is called the characteristic line field of $\mathcal{D}$, and integral curves of $\mathcal{L}(\mathcal{D})$ are called characteristic leaves of $\mathcal{D}$.

In [5], Golubev shows that if $\mathcal{D}_{t}, 0 \leqslant t \leqslant 1$, is a smooth family of oriented Engel structures on a closed oriented 4-manifold $M$ such that $\mathcal{L}\left(\mathcal{D}_{t}\right)=\mathcal{L}\left(\mathcal{D}_{0}\right)$ for all $t$, then there exists a smooth family of diffeomorphisms $\varphi_{t}$ of $M$ such that $\left(\varphi_{t}\right)_{*} \mathcal{D}_{0}=\mathcal{D}_{t}$ for all $t$. Thus if we want to investigate perturbations of Engel structures it is essential to understand the behaviour of the associated characteristic line fields.

The most naive question on this line may be the following.

Question. Given a line field $\mathcal{L}$ on a 4-manifold $M$, can one find an Engel structure $\mathcal{D}$ whose characteristic line field coincides with $\mathcal{L}$ ?

There are some necessary conditions that $\mathcal{L}$ should satisfy: The normal bundle of $\mathcal{L}$ must be trivial and $\mathcal{L}$ must admit both a transverse contact structure and a leafwise projective structure ([2, Proposition 3.4], [8]). But the complete answer is not known. (See the introduction of [9].)

In [3], Gershkovich studied various properties of Engel structures and constructed many examples. Then he asked, among others, if there exists an Engel structure on $\mathbf{R}^{4}$ with a compact characteristic leaf. The main purpose of this paper is to give the following.

TheOREM 1. There exists an Engel structure on $\mathbf{R}^{4}$ which admits a compact characteristic leaf.

We shall also construct analogous examples on other open 4-manifolds. (See Theorem 2 in Section 4 for the precise statement.)

\section{Construction ON $\mathbf{R}^{4}$}

We shall prove Theorem 1 by an explicit construction.

First of all we recall a basic method of constructing an Engel structure. Let $N$ be a 3-manifold and $\mathcal{E}$ a paralellisable contact structure on $N$. This means that there exist two vector fields $\xi$ and $\eta$ on $N$ such that $\mathcal{E}$ is spanned by them at each point of $N$. Then we construct an Engel structure on $N \times \mathbf{R}$ (or on $N \times S^{1}$ ) as follows: Define vector fields on $N \times \mathbf{R}$ (or on $N \times S^{1}$ ) by $X=\frac{\partial}{\partial \theta}$ and $Y=(\cos \theta) \xi+(\sin \theta) \eta\left(\theta \in \mathbf{R}\right.$ or $\left.S^{1}\right)$. Let $\mathcal{D}=\mathcal{D}(\xi, \eta)$ denote the 2-plane field spanned by $X$ and $Y$. Then one can easily check that $\mathcal{D}$ is an Engel structure. Moreover, one sees that the characteristic line field $\mathcal{L}(\mathcal{D})$ of this Engel structure is spanned by $X$. For convenience, hereafter we call $\mathcal{D}(\xi, \eta)$ the Engel structure obtained from $(\xi, \eta)$ by the basic method.

Now we shall construct a desired Engel structure on $\mathbf{R}^{4}$ by gluing three pieces together. 
The first piece $\left(A, \mathcal{D}_{A}\right)$ is simply defined as follows: Let $A=D^{2} \times[-1,1] \times S^{1}$ $=\left\{(x, y, z, \theta) \in \mathbf{R}^{3} \times S^{1} \mid x^{2}+y^{2} \leqslant 1,-1 \leqslant z \leqslant 1\right\}$, and give on it an Engel structure by the basic method. Namely, put $\xi_{0}=\frac{\partial}{\partial x}+z \frac{\partial}{\partial y}$ and $\eta_{0}=\frac{\partial}{\partial z}$ for $(x, y, z) \in \mathbf{R}^{3}$. Then $\xi_{0}$ and $\eta_{0}$ span a standard contact structure $\mathcal{E}_{0}$ on $D^{2} \times[-1,1]$. We define the Engel structure $\mathcal{D}_{A}$ on $A$ by $\mathcal{D}_{A}=\mathcal{D}\left(\xi_{0}, \eta_{0}\right)$. Here we make an obvious remark that all the characteristic leaves of $\mathcal{D}_{A}$ are compact.

The second piece $\left(B, \mathcal{D}_{B}\right)$ is also quite simple. Let $\mathcal{D}_{0}$ be an Engel structure on $\mathbf{R}^{4}$ defined by $\mathcal{D}_{0}=\mathcal{D}\left(\xi_{0}, \eta_{0}\right)$, where $\xi_{0}$ and $\eta_{0}$ are as above. Then, we dig a hole $H=\left\{(x, y, z, \theta) \in \mathbf{R}^{4} \mid x^{2}+y^{2}<4,-2<z<2, \theta>-1\right\}$ in $\mathbf{R}^{4}$, and define $B$ by $B=\mathbf{R}^{4}-H$ and $\mathcal{D}_{B}$ to be the restriction of $\mathcal{D}_{0}$ to $B$.

The third piece $\left(C, \mathcal{D}_{C}\right)$ is constructed as follows: Set $C=\left\{(x, y, z, \theta) \in \mathbf{R}^{3} \times S^{1} \mid\right.$ $\left.1 \leqslant x^{2}+y^{2} \leqslant 4,-1 \leqslant z \leqslant 1,-\varepsilon \leqslant \theta \leqslant \varepsilon(\bmod 2 \pi)\right\}$, where $\varepsilon$ is a small positive number. We shall construct an Engel structure $\mathcal{D}_{C}$ by modifying the one obtained by the basic method. Denote by $(\rho, \varphi)$ the polar coordinates for $(x, y)$. Namely, $x=\rho \cos \varphi$ and $y=\rho \sin \varphi$. Let $\lambda$ be a smooth function defined on the closed interval $1 \leqslant \rho \leqslant 2$ such that $\lambda(\rho) \equiv 0$ near $\rho=1$, that $\lambda(\rho) \equiv 1$ near $\rho=2$, and that $0 \leqslant \lambda(\rho) \leqslant 1$ for all $\rho$. Let $\Phi: C \rightarrow\left\{(x, y, z, \theta) \in \mathbf{R}^{3} \times S^{1} \mid 1 \leqslant x^{2}+y^{2}\right.$ $\leqslant 4,-1 \leqslant z \leqslant 1\}$ be a map defined by $\Phi(\rho \cos \varphi, \rho \sin \varphi, z, \theta)=(\rho \cos \varphi, \rho \sin \varphi, z, \theta+\varphi)$. Now, following the basic method, set $X=\frac{\partial}{\partial \theta}$ and $Y=(\cos \theta) \xi_{0}+(\sin \theta) \eta_{0}$, where $\xi_{0}$ and $\eta_{0}$ are the vector fields defined above. Then we modify $Y$ and make a new vector field $\widehat{Y}=\lambda(\rho) Y+(1-\lambda(\rho))\left(\left.\Phi^{-1}\right|_{\Phi(C)}\right)_{*} Y$. Our plane field $\mathcal{D}_{C}$ is defined to be the one spanned by $X$ and $\widehat{Y}$.

LEMma 1. We have the following.

(1) $\mathcal{D}_{C}$ is an Engel structure.

(2) $\widehat{Y}=Y$ near $\rho=2$.

(3) $\Phi_{*} \widehat{Y}=Y$ near $\rho=1$.

Proof: (2) and (3) are obvious. Let us prove (1). A direct computation shows that

$$
\begin{aligned}
\hat{Y}=\{\lambda(\rho) \cos \theta+(1-\lambda(\rho)) \cos (\theta+\varphi)\} \xi_{0} & +\{\lambda(\rho) \sin \theta+(1-\lambda(\rho)) \sin (\theta+\varphi)\} \eta_{0} \\
& +\frac{1-\lambda(\rho)}{\rho} \cos (\theta+\varphi)(\sin \varphi-z \cos \varphi) \frac{\partial}{\partial \theta}
\end{aligned}
$$

We put $\widehat{Z}=[X, \widehat{Y}]$. Then, one can easily check that $L(X, \widehat{Y}, \widehat{Z})=L\left(\xi_{0}, \eta_{0}, \frac{\partial}{\partial \theta}\right)$ at every point of $C$, where $L(\quad)$ means the linear span. Moreover, we see that $[\widehat{Y}, \widehat{Z}]$ does not belong to $L(X, \widehat{Y}, \widehat{Z})$ at any point of $C$. In fact, a direct computation shows that

$$
[\widehat{Y}, \widehat{Z}] \equiv\left\{\lambda(\rho)^{2}+(1-\lambda(\rho))^{2}+2 \lambda(\rho)(1-\lambda(\rho)) \cos \varphi\right\}\left[\xi_{0}, \eta_{0}\right]
$$


$\left(\bmod \xi_{0}, \eta_{0}, \frac{\partial}{\partial \theta}\right)$, and the right hand side of this equality never vanishes. This implies that $\mathcal{D}_{C}$ is an Engel structure, completing the proof.

Now we shall glue these three pieces together. Denote the two connected components of $\partial C$ by $\partial_{1} C=\{\rho=1\}$ and $\partial_{2} C=\{\rho=2\}$. First, we paste $C$ and $B$ by $\iota$, where $\iota: \partial_{2} C \rightarrow \partial B$ is the inclusion map. Next, paste $C$ and $A$ by $\Phi$, where we regard $\Phi$ as the map $\Phi: \partial_{1} C \rightarrow \partial A$ defined by $\Phi(\cos \varphi, \sin \varphi, z, \theta)=(\cos \varphi, \sin \varphi, z, \theta+\varphi)$. Denote the resulting space by $W$ :

$$
W=A \bigcup_{\Phi} C \bigcup_{\iota} B
$$

Then, one can check that the Engel structures on the pieces match and produce a welldefined Engel structure on $W$. Obviously, the characteristic line field of this Engel structure has compact leaves in $A$. Finally, take the interior of $W$ and denote it by $M$. Then, $M$ is diffeomorphic to $\mathbf{R}^{4}$. In fact, this can be seen as follows: Let $\gamma$ and $\delta$ be, respectively, the longitudinal loop (that is, the loop defined by $\varphi=0$ ) and the diagonal loop (that is, the loop defined by $\varphi=\theta$ ) of $\partial D^{2} \times S^{1}$, where $\varphi \in \partial D^{2}$ and $\theta \in S^{1}$ are the angular coordinates. Then, there exists a diffeomorphism $h$ of $D^{2} \times S^{1}$ to itself such that

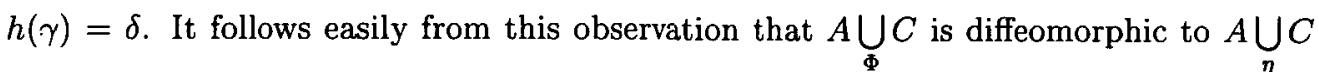
(the manifold obtained from $A$ and $C$ by pasting $\partial_{1} C$ to $\partial A$ via $\eta$ ), where $\eta: \partial_{1} C \rightarrow \partial A$ is the map defined by $\eta(\cos \varphi, \sin \varphi, z, \theta)=(\cos (-\theta), \sin (-\theta), z, \varphi)$. Thus we have

$$
M=\operatorname{Int}\left(A \bigcup_{\Phi} C \bigcup_{\iota} B\right) \approx \operatorname{Int}\left(A \bigcup_{\eta} C \bigcup_{\iota} B\right) \approx \operatorname{Int} B \approx \mathbf{R}^{4}
$$

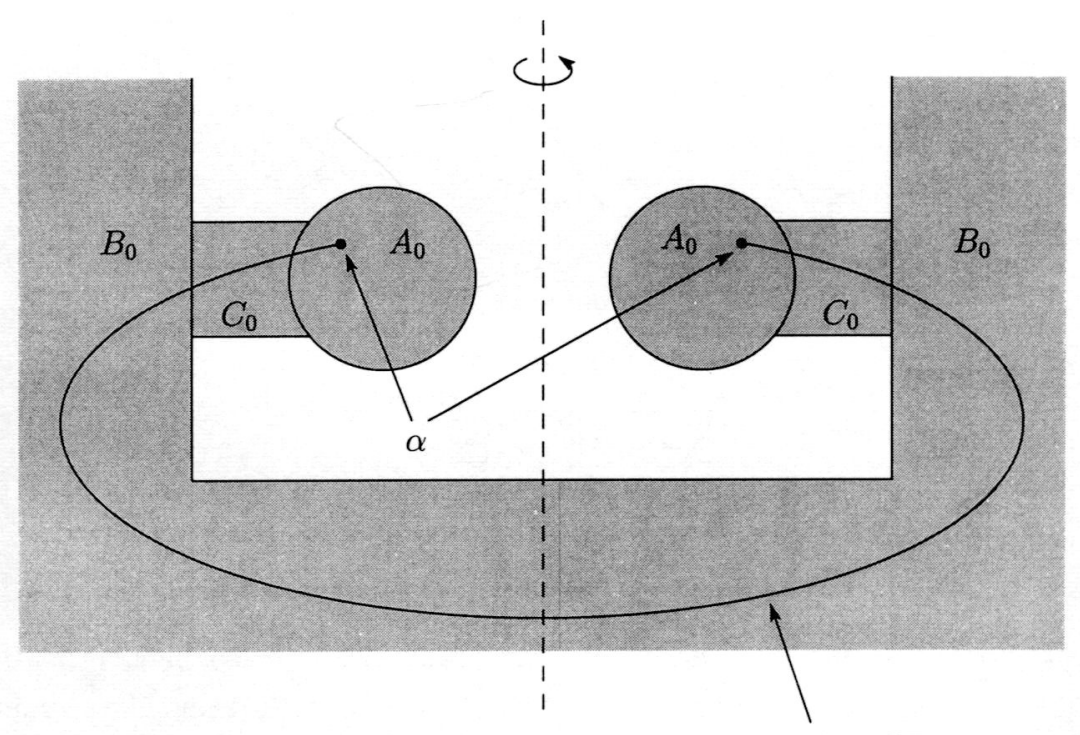

a disk $D$ such that $\partial D=\alpha$

Figure 1 
as desired, where $\approx$ means diffeomorphic. The Engel structure induced on $M$ has a compact characteristic leaf in $\operatorname{Int} A$. This completes the construction and Theorem 1 is established.

Following the referee's advice, we would like to give here a picture (Figure 1) for visualisation of our manifold $M$. Let $\alpha=\left\{((1-\varepsilon) \cos \theta,(1-\varepsilon) \sin \theta, 0, \theta) \mid \theta \in S^{1}\right\} \subset A$, where $0<\varepsilon<1$. The figure indicates how $\alpha$ bounds a disk in $M$. Here we put $A_{0}=A \cap\left(\mathbf{R}^{2} \times\{0\} \times S^{1}\right), B_{0}=B \cap\left(\mathbf{R}^{2} \times\{0\} \times \mathbf{R}\right)$ and $C_{0}=C \cap\left(\mathbf{R}^{2} \times\{0\} \times \mathbf{R}\right)$.

\section{Construction ON OTHER OPEN MANIFOldS}

In this section, we shall show that the same construction as in the previous section can in fact be applied to modifying any Engel structure on any open 4-manifold to create a compact characteristic leaf. Here, by an open manifold we mean a $C^{\infty}$ non-compact (paracompact, Hausdorff) manifold without boundary. We have the following.

THEOREM 2. Let $M$ be an open 4-manifold and $\mathcal{D}$ an Engel structure on $M$. Then one can modify $\mathcal{D}$ to obtain a new Engel structure on $M$ whose characteristic line field has a compact leaf.

Proof: Let $M$ and $\mathcal{D}$ be as above. Take an arbitrary point $p$ of $M$. Then, since the Darboux theorem holds for Engel structures (see [1]), we can find a compact neighbourhood $N$ of $p$ such that there exists a diffeomorphism $\psi:\{(x, y, z, \theta)$ $\left.\in \mathbf{R}^{4} \mid x^{2}+y^{2} \leqslant 4,-2 \leqslant z \leqslant 2,-1 \leqslant \theta \leqslant 1\right\} \rightarrow N$ and that the Engel structure $\mathcal{D}$ restricted to $N$ is just $\psi_{*} \mathcal{D}\left(\xi_{0}, \eta_{0}\right)$, where $\mathcal{D}\left(\xi_{0}, \eta_{0}\right)$ is the Engel structure defined in Section 3. Next, we shall take a smooth proper simple curve $\gamma:[0, \infty) \rightarrow M$ such that $\gamma(0)=\psi(0,0,0,1)$ and that $\gamma((0, \infty)) \subset M-N$, where proper means, as usual, that for each compact set $K$ of $M$ the set $\gamma^{-1}(K)$ is also compact. Such a curve can be constructed, for instance, in the following way. Take a Morse function $f$ on $M$ such that $f^{-1}((-\infty, t])$ is compact for all $t \in \mathbf{R}$ (see [7, Corollary 6.7]) such that every non-negative integer is a regular value of $f$, that $f(\gamma(0))=0$, and that $N \subset f^{-1}((-\infty, 0])$. Let $\left\{U_{n}\right\}$ be a decreasing sequence of codimension 0 submanifolds of $M$ such that $U_{n}$ is a non-compact connected component of $f^{-1}([n, \infty))$ for each $n$. Then, one can construct a simple smooth curve $\gamma_{n}:[n, n+1] \rightarrow U_{n}-\operatorname{Int} U_{n+1}$ such that $\gamma_{n}(n) \in \partial U_{n}, \gamma_{n}(n+1) \in \partial U_{n+1}$ and $\gamma_{n}(n+1)=\gamma_{n+1}(n+1)$, and that the combined curve $\gamma_{n} * \gamma_{n+1}:[n, n+2] \rightarrow U_{n}-\operatorname{Int} U_{n+2}$ is still simple and smooth. Now, one can define a desired curve $\gamma:[0, \infty) \rightarrow M$ by setting $\gamma(t)=\gamma_{n}(t)$ for $t \in[n, n+1]$. We then take a smooth proper embedding $j: D^{3} \times[0, \infty) \rightarrow M$ such that $j \mid(\{0\} \times[0, \infty))=\gamma$ and that $j \mid\left(D^{3} \times[0, \infty)\right) \cap N=j\left(D^{3} \times\{0\}\right) \subset \psi\left(\left\{(x, y, z, 1) \mid x^{2}+y^{2} \leqslant 4,-2 \leqslant z \leqslant 2\right\}\right)$. (Such an embedding can be constructed by considering a tubular neighbourhood of the image of $\gamma$.) 
Now, let $H$ be the interior of the union of $N$ and the image of $j$, and put $B=M-H$. Denote by $\mathcal{D}_{B}$ the restriction of $\mathcal{D}$ to $B$. The rest of the construction is the same as in the previous section. Let $\left(A, \mathcal{D}_{A}\right)$ and $\left(C, \mathcal{D}_{C}\right)$ be the pieces defined in Section 3. By pasting $A, B$ and $C$ via $\Phi: \partial_{1} C \rightarrow \partial A$ and $\iota: \partial_{2} C \rightarrow \partial N(\subset \partial B)$ just as in Section 3 , and taking the interior of the resulting manifold, we obtain an open manifold $\widehat{M}$ diffeomorphic to the original manifold $M$, and the Engel structure of $\widehat{M}$ induced from those of $A, B$ and $C$ admits a compact characteristic leaf. Theorem 2 is proven.

\section{REMARKS}

REMARK 1. Compact characteristic leaves of Engel structures constructed in this paper are all null-homotopic. Thus, we have actually shown that every open 4-manifold admitting an Engel structure admits an Engel structure with null-homotopic compact characteristic leaf. The author does not know if the same is true for compact 4-manifolds. The only one example of a compact Engel manifold with null-homotopic characteristic leaves the author knows so far is the following. (This is communicated to him by Y. Mitsumatsu.) Let $\mathcal{E}^{\prime}$ be a contact structure on $S^{2} \times S^{1}$ which is homotopic to $\mathcal{H} \oplus 0$, where $\mathcal{H}$ is a vector bundle on $S^{2}$ whose unit circle bundle is diffeomorphic to $S^{3}$. The existence of such a contact structure is guaranteed by Lutz' existence theorem for contact structures ([6]). Take a prolongation (see [8]) of $\mathcal{E}^{\prime}$ and a fiberwise double cover $\widehat{\mathbf{P E}} \mathcal{E}^{\prime}$ of the projectivisation $\mathbf{P} \mathcal{E}^{\prime}$ of $\mathcal{E}^{\prime}$. Then we obtain an Engel structure on $\widetilde{\mathbf{P} \mathcal{E}^{\prime}}$, whose characteristic leaves are all null-homotopic circles, as desired.

REMARK 2. (We use the notation in Section 3.) By Montgomery's deformation theorem in [8, Theorem 4], we can modify our examples so that the resulting characteristic line fields have various holonomies (Poincaré maps): Let $h$ be an arbitrary contact diffeomorphism of the standard contact manifold $\left(D^{2} \times[-1,1], \mathcal{E}_{0}\right)$ which is $C^{\infty}$-near the identity and is the identity near the boundary $\partial\left(D^{2} \times[-1,1]\right)$. Then by Montgomery's theorem we obtain an Engel structure $\widetilde{\mathcal{D}_{A}}$ on $A=D^{2} \times[-1,1] \times S^{1}$ such that $\mathcal{L}\left(\widetilde{\mathcal{D}_{A}}\right)$ is transverse to $D^{2} \times[-1,1] \times\{\theta\}$ for each $\theta \in S^{1}$, and that the holonomy of $\mathcal{L}\left(\widetilde{\mathcal{D}_{A}}\right)$ coincides with $h$. Since $\widetilde{\mathcal{D}_{A}}=\mathcal{D}_{A}$ near $\partial A$, in the construction of the previous sections we can replace the piece $\left(A, \mathcal{D}_{A}\right)$ with the new piece $\left(A, \widetilde{\mathcal{D}_{A}}\right)$ and thus obtain various, mutually distinct examples.

\section{REFERENCES}

[1] R.L. Bryant, S.S. Chern, R.B. Gardner, H.L. Goldschmidt and P.A. Griffiths, Exterior differential systems, Math. Sci. Res. Inst. Publ. 18 (Springer Verlag, New York, 1991).

[2] R.L. Bryant and L. Hsu, 'Rigidity of integral curves of rank 2 distributions', Invent. Math. 114 (1993), 435-461.

[3] V. Gershkovich, 'Engel structures on four dimensional manifolds', (preprint, Univ, of Melbourne, 1993). 
[4] V. Gershkovich, 'Exotic Engel structures on $\mathbf{R}^{4}$, Russian J. Math. Phys. 3 (1995), 207-226.

[5] A. Golubev, 'On the stability of maximally nonholonomic two-plane fields in four dimensions', Internat. Math. Res. Notices 11 (1997), 523-529.

[6] R. Lutz, 'Structures de contact sur les fibres principaux en cercles de dimension trois', Ann. Inst. Fourier (Grenoble) 27 (1977), 1-15.

[7] J. Milnor, Morse theory, Ann. of Math. Studies 51 (Princeton Univ. Press, Princeton, N. J., 1963).

[8] R. Montgomery, 'Engel deformations and contact structures', in Northern California symplectic geometry seminar, Amer. Math. Soc. Trans. Ser. 2196 (Amer. Math. Soc., Providence, R.I., 1999), pp. 103-117.

[9] R. Montgomery and M. Zhitomirskii, 'Geometric approach to Goursat flags', Ann. Inst. H. Poincaré Anal. Non Linéaire 18 (2001), 459-493.

Division of Mathematical Sciences and Physics

Graduate School of Science and Technology

Chiba University

1-33 Yayoicho, Inage-ku

Chiba 263-8522

Japan

e-mail: inaba@math.s.chiba-u.ac.jp 